\title{
Adult-onset autoimmune diabetes: comparative analysis of classical and latent presentation
}

Lúcia Fadiga ${ }^{* *} \mathbb{0}$, Joana Saraiva ${ }^{1,2}$, Diana Catarino', João Frade ${ }^{3}$, Miguel Melo ${ }^{1,2,4 \dagger}$ and Isabel Paiva ${ }^{1 \dagger}$

\begin{abstract}
Introduction: Adult-onset autoimmune diabetes (AID) has two different phenotypes: classic type 1 diabetes mellitus (T1DM), with insulin requirement just after diagnosis, and latent autoimmune diabetes in adults (LADA). The purpose of this study is to characterize patients with AID followed on a tertiary centre, comparing classic T1DM and LADA.

Methods: We collected data from patients with diabetes and positive islet autoantibodies, aged 30 years old and over at diagnosis. Patients who started insulin in the first 6 months were classified as T1DM and patients with no insulin requirements in the first 6 months were classified as LADA. Data regarding clinical presentation, autoantibodies, A1C and C-peptide at diagnosis, pharmacologic treatment and complications were analysed.

Results: We included 92 patients, 46 with classic T1DM and 46 with LADA. The percentage of females was 50\% in T1DM group and $52.1 \%$ in LADA group. The median age at diagnosis was 38 years (IQR-15) for T1DM and 42 years (IQR-15) for LADA ( $p=0.057)$. The median time between diagnosis of diabetes and diagnosis of autoimmune aetiology was 0 months in T1DM group and 60 months in LADA group $(p<0.001)$. The mean BMl at diagnosis was $24.1 \mathrm{~kg} /$ $\mathrm{m}^{2}$ in T1DM group and $26.1 \mathrm{~kg} / \mathrm{m}^{2}$ in LADA group ( $\left.p=0.042\right)$. In T1DM group, 67.4\% of the patients had more than one positive autoantibody, comparing to $41.3 \%$ of LADA patients $(p=0.012)$. There was no statistical difference in what concerns to title of GAD autoantibodies, A1C and C-peptide at diagnosis of autoimmune aetiology. The presence of symptoms at diagnosis was associated with T1DM group $(p<0.001)$. The median daily insulin dose was $40 \mathrm{IU}$ for T1DM $(0.58 \mathrm{IU} / \mathrm{kg})$ and $33.5 \mathrm{IU}$ for LADA $(0.57 \mathrm{IU} / \mathrm{kg})$, with no statistical difference. LADA patients were more often under non-insulin antidiabetic drugs $(p=0.001)$. At 10 years follow up, $21.1 \%$ of T1DM patients and $63.3 \%$ of LADA patients had microvascular complications ( $p=0.004$ ). Diabetic nephropathy was present in 23.5\% of T1DM patients and $53.3 \%$ of LADA patients $(p=0.047)$. At the last evaluation, $55.6 \%$ of T1DM and $82.6 \%$ of LADA patients had metabolic syndrome and this difference was independent of diabetes duration.
\end{abstract}

Conclusion: Patients with classic T1DM presented more often with symptoms, lower BMI and higher number of autoantibodies, which may be related to a more aggressive autoimmune process. Patients with LADA developed more frequently microvascular complications for the same disease duration, namely diabetic nephropathy, and had more often metabolic syndrome.

Keywords: Autoimmune disease, Diabetes complications, Diabetes mellitus, type 1, Latent autoimmune diabetes in adults, Insulin resistance

\footnotetext{
*Correspondence: Imsfadiga@gmail.com

${ }^{\dagger}$ Miguel Melo and Isabel Paiva contributed equally to the work

${ }^{1}$ Endocrinology, Diabetes and Metabolism Department, Centro Hospitalar e Universitário de Coimbra, Coimbra, Portugal

Full list of author information is available at the end of the article
}

\begin{abstract}
Introduction
Adult-onset autoimmune diabetes (AID) is a complex and heterogeneous condition, whose main feature is the presence of serum diabetes-related autoantibodies $[1,2]$.
\end{abstract}

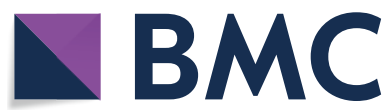

(c) The Author(s) 2020. This article is licensed under a Creative Commons Attribution 4.0 International License, which permits use, sharing, adaptation, distribution and reproduction in any medium or format, as long as you give appropriate credit to the original author(s) and the source, provide a link to the Creative Commons licence, and indicate if changes were made. The images or other third party material in this article are included in the article's Creative Commons licence, unless indicated otherwise in a credit line to the material. If material is not included in the article's Creative Commons licence and your intended use is not permitted by statutory regulation or exceeds the permitted use, you will need to obtain permission directly from the copyright holder. To view a copy of this licence, visit http://creativeco mmons.org/licenses/by/4.0/. The Creative Commons Public Domain Dedication waiver (http://creativecommons.org/publicdomain/ zero/1.0/) applies to the data made available in this article, unless otherwise stated in a credit line to the data. 
AID may present as a sudden onset of insulin deficiency, with symptoms and frequent ketosis, being the patient dependent on exogenous insulin just after the diagnosisthe so-called "classic type 1 diabetes mellitus" (T1DM). On the other hand, patients may present with slowly progressive insulin deficiency, variable levels of insulin resistance and often do not require insulin treatment for a considerable period after diagnosis-the so-called "latent autoimmune diabetes in adults" (LADA) [1, 3, 4]. According to the Immunology of Diabetes Society (IDS), LADA diagnosis is based on three criteria: a minimal age of 30 years at diabetes onset, the presence of circulating islet autoantibodies and lack of insulin requirement for at least 6 months after diagnosis [4].

Although LADA and T1DM share common genetic and immune characteristics, LADA patients also seem to match some features of type 2 diabetes (T2DM), like being often overweight/obese, physically inactive and having other criteria for the metabolic syndrome, which leads to insulin resistance $[1,3,5]$. In fact, both autoimmunity and insulin resistance play important roles in the pathogenesis of LADA and, depending on the predominant factor, the phenotype will be more T1DM-like or T2DM-like $[1,6]$.

The purpose of this study is to characterize patients with AID, comparing classic T1DM and LADA in what concerns to clinical presentation, metabolic control, pharmacologic treatment and diabetic complications.

\section{Methods}

We conducted a retrospective cohort study at our tertiary care centre, including patients with diabetes mellitus (DM) and one or more positive diabetes-related autoantibodies-glutamic acid decarboxylase antibodies (GADA), islet cell antibodies (ICA), tyrosine phosphatase-like insulinoma antigen 2 antibodies (IA2) and endogenous insulin antibodies (IAA) - in blood samples analysed between the 1st January 2007 and the 30th June 2017 at our laboratory. ICA were analysed through indirect immunofluorescence (Mago $4^{\circledR}$ ), a semi-quantitative method. GADA, IA2 and IAA were analysed through radioimmunoassay (Wallac Wizard $1470^{\circledR}$ Automatic Gamma Counter), with reference values of $1.0 \mathrm{U} / \mathrm{mL}, 1.0$ $\mathrm{U} / \mathrm{mL}$ and $0.4 \mathrm{U} / \mathrm{mL}$, respectively. We excluded patients with diabetes onset before 30 years of age (median age 25 years, IQR 5) and with relevant lack of clinical data, such as age of onset of diabetes or age of onset of insulin therapy (Fig. 1).

Patients who started insulin in the first 6 months after diabetes diagnosis were included in the T1DM group and patients with no insulin requirement in the first 6 months after diagnosis were included in the LADA group.

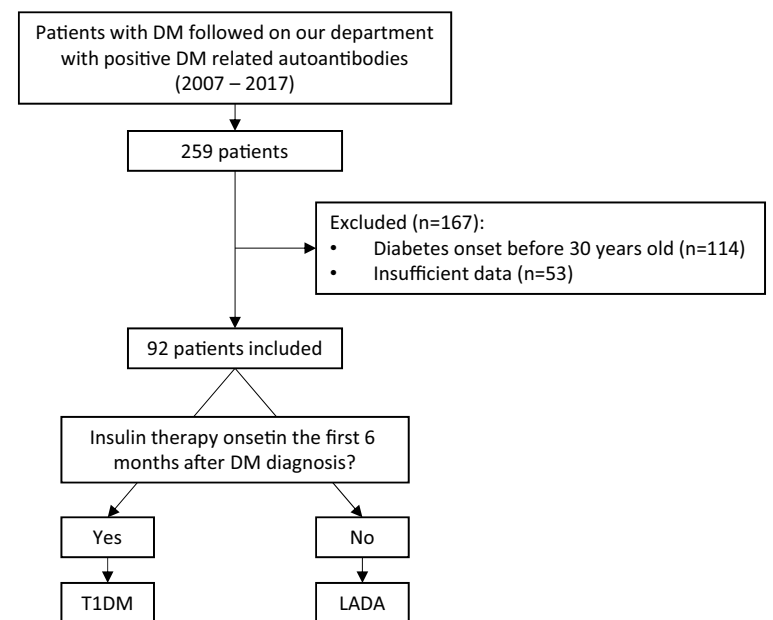

Fig. 1 Representation of the study methodology

We reviewed patients' clinical records and collected data regarding age, sex, weight, height, body mass index (BMI), date of diagnosis of diabetes, clinical presentation, haemoglobin A1c (A1C) and fasting C-peptide (C-pep) at diagnosis, date of autoantibodies measurement and autoantibodies levels.

All patients were evaluated at the last medical appointment in what concerns to autoimmune comorbidities, current pharmacologic treatment, hypertension, dyslipidaemia and metabolic syndrome. For the majority of autoimmune diseases, the screening was clinical or based in abnormal blood tests findings during follow-up (e.g. macrocytic anaemia for atrophic gastritis or abnormal liver function tests for autoimmune hepatitis); autoimmune thyroiditis was screened at least once a year through the analysis of thyroid antibodies.

Metabolic syndrome (MetS) was considered to be present if patients had 2 of 4 of the following criteria: hypertension, obesity, elevated triglycerides and low high-density lipoprotein cholesterol (or lipid-lowering drugs) [7]. Patients with at least 10 years of diabetes duration were assessed for diabetic complications at the mark of 10 years. Diabetic nephropathy was considered based on estimated glomerular filtration rate (GFR) and albuminuria, according to KDIGO 2012 guidelines [8]. The remaining complications were considered based on clinical records, including yearly evaluation at the Department of Ophthalmology.

Statistical analysis was performed using IBM SPSS Statistics v.23 for Windows. Normally distributed data are given as mean and standard deviation. Non-normally distributed data are given as median and range. Differences between groups were evaluated using independentsamples T-test and Mann-Whitney test and association 
between variables was assessed with Chi square test and Fisher's exact test. A $p<0.05$ value was considered significant.

\section{Results}

We included 92 patients, 46 with T1DM and 46 with LADA. The descriptive characteristics of the patients, including anthropometric, clinical and biochemical data, as well as comparative analysis between both groups, are presented in Table 1.

The proportion of female patients in both groups was similar. The median age at diabetes diagnosis was 38 years for T1DM (range 30-65 years, IQR 15) and 42 years for LADA (range 30-76, IQR 15). The median time between diabetes diagnosis and starting insulin therapy (which was the discriminative criteria of patients in the two groups) was 0 months in T1DM (range $0-4$ months, IQR 0.0 ) and 48 months in LADA (range 6-396 months, IQR 56.3).

The recognition of the autoimmune aetiology for diabetes was defined as the time of measurement of diabetes-related autoantibodies. The median interval time between both diagnoses was 0 months in T1DM (range
0-216 months, IQR 3.0) and 60 months in LADA (range $0-444$ months, IQR 92.3), $p<0.001$. Included patients were followed for a median of 8 years for T1DM group (range 0.1-29.0 years, IQR 7.5) and 11 years for LADA group (range 1.0-43.0 years, IQR 10.5).

At the diagnosis of diabetes, $87 \%$ of T1DM patients and $45.7 \%$ of LADA patients presented with symptoms $(p<0.001)$. Among these, two patients with T1DM presented with diabetic ketoacidosis, and all the remaining patients presented with polyuric/polydipsic syndrome.

At the diagnosis of the autoimmune aetiology, T1DM group had a mean BMI in the normal range (mean $24.1 \pm 3.8 \mathrm{~kg} / \mathrm{m}^{2}$ ) and lower than the mean BMI in the LADA group, which was in the range of overweight (mean $26.1 \pm 5.2 \mathrm{~kg} / \mathrm{m}^{2}$ ); the latter difference was statistically significant $(p=0.042)$. At this point, T1DM had numerically (but not statistically) higher A1C and lower C-peptide.

In what concerns to the number of positive diabetesrelated autoantibodies, T1DM patients had a median of 2 positive antibodies and LADA patients had a median of 1 positive antibody $(p=0.013)$. In T1DM group, $67.4 \%$ of the patients had more than one positive autoantibody,

Table 1 Clinical and biochemical characteristics of included patients

\begin{tabular}{|c|c|c|c|}
\hline & T1DM $(n=46)$ & $\operatorname{LADA}(n=46)$ & $p$ \\
\hline Females, n (\%) & $23(50.0 \%)$ & $24(52.2 \%)$ & 0.835 \\
\hline Age at diabetes diagnosis, median (IQR) & $38(15)$ & $42(15)$ & 0.057 \\
\hline $\begin{array}{l}\text { Months between diagnosis of diabetes and insulin therapy beginning, } \\
\text { median (IQR) }\end{array}$ & $0(0.0)$ & $48(56.3)$ & $<0.001^{*}$ \\
\hline $\begin{array}{l}\text { Months between diagnosis of diabetes and diagnosis of autoimmune aetiol- } \\
\text { ogy, median (IQR) }\end{array}$ & $0(3.0)$ & $60(92.3)$ & $<0.001^{*}$ \\
\hline Diabetes duration at the last follow-up, years, median (IQR) & $8.0(7.5)$ & $11.0(10.5)$ & $0.023^{*}$ \\
\hline \multicolumn{4}{|l|}{ At the diagnosis of diabetes } \\
\hline Symptoms at presentation, n (\%) & $40(87.0 \%)$ & $21(45.7 \%)$ & $<0.001^{* *}$ \\
\hline Diabetic ketoacidosis, n & 2 & 0 & \\
\hline Polyuric/polydipsic syndrome, n & 38 & 21 & \\
\hline \multicolumn{4}{|l|}{ At the measurement of diabetes-related autoantibodies } \\
\hline BMI $\left(\mathrm{kg} / \mathrm{m}^{2}\right)$, mean $( \pm \mathrm{SD})$ & $24.1( \pm 3.8)$ & $26.1( \pm 5.2)$ & $0.042^{* * *}$ \\
\hline $\mathrm{A} 1 \mathrm{C}(\%)$, mean $( \pm \mathrm{SD})$ & $10.3( \pm 2.4)$ & $9.5( \pm 2.2)$ & 0.113 \\
\hline C-peptide (ng/mL), median (IQR) & $0.7(0.6)$ & $1.0(1.4)$ & 0.152 \\
\hline Number of positive antibodies, median (IQR) & $2(2.0)$ & $1(1.0)$ & $0.013^{*}$ \\
\hline Patients with > 1 positive antibody, n (\%) & $31(67.4 \%)$ & $19(41.3 \%)$ & $0.012^{* *}$ \\
\hline Positive GADA, n/total (\%) & $43 / 46(93.5 \%)$ & $39 / 46(84.8 \%)$ & 0.180 \\
\hline GADA title of patients with positive GADA (U/mL), median (IQR) & $21.4(94.1)$ & $11.9(60.4)$ & 0.229 \\
\hline Positive ICA, n/total (\%) & $30 / 43(69.8 \%)$ & $23 / 38(60.5 \%)$ & 0.383 \\
\hline Positive IA2, n/total (\%) & $17 / 43(39.5 \%)$ & $10 / 45(22.2 \%)$ & 0.078 \\
\hline IA2 title of patients with positive IA2 (U/mL), median (IQR) & $10.5(31.1)$ & $4.1(10.8)$ & 0.093 \\
\hline Positive IAA, n/total (\%) & $4 / 38(10.5 \%)$ & $1 / 35(2.9 \%)$ & 0.359 \\
\hline
\end{tabular}

* Independent-samples Mann-Whitney test

** Chi square test

*** Independent-samples T-test 
comparing to $41.3 \%$ of LADA patients $(p=0.012)$. GADA was the most prevalent positive autoantibody in both groups. The number of patients with positive GADA, ICA, IA2 and IAA was numerically higher in T1DM group comparing to LADA group and the titles of GADA and IA2 were higher in T1DM (not statistically significant).

In what concerns to the therapeutic regimen on the last follow-up (Table 2), all the patients in T1DM group were treated with insulin. Four patients in LADA group remained insulin-free. These patients had a diabetes duration from 4 to 10 years, were all under metformin and dipeptidyl peptidase-4 inhibitors (DPP4-i) and one was also under a sulfonylurea. At the last visit, their A1C values were $6.4 \%, 7.3 \%, 7.5 \%$ and $8.3 \%$.

A higher proportion of patients with T1DM were under basal-bolus insulin therapy: $72.7 \%$ were under basalbolus therapy with multiple daily injections (MDI) comparing to $58.6 \%$ on LADA group; 2 patients with T1DM and 1 patient with LADA were under continuous subcutaneous insulin infusion (CSII). Remarkably, three T1DM patients were under basal insulin only; these patients had a short duration of disease (between 1 and 2 years), and A1C on last follow-up was $5.5 \%, 5.7 \%$ and $7.4 \%$.

The median total insulin daily dose (TDD) was $40.0 \mathrm{IU}$ in T1DM group and 33.5 IU in LADA group, which corresponded to $0.58 \mathrm{IU} / \mathrm{kg}$ in T1DM and $0.57 \mathrm{IU} / \mathrm{kg}$ in LADA. There were no statistically significant differences in TDD, before and after adjustment for body weight.

Non insulin drugs were more often used in LADA patients: $54.3 \%$ comparing to $20.5 \%$ in $\operatorname{T1DM}(p=0.001)$.
Metformin was prescribed to $47.8 \%$ of LADA patients and $15.9 \%$ of T1DM patients $(p=0.001)$; DPP4-i were prescribed to $37 \%$ of LADA patients, comparing to $11.4 \%$ of T1DM patients $(p=0.005)$. No T1DM patient was under sulfonylureas, glucagon-like peptide-1 receptor agonists (GLP1-ra) or sodium-glucose co-transporter-2 inhibitors (SGLT2i), which were prescribed in a minority of LADA patients.

Table 3 summarizes data concerning autoimmune (AI) comorbidities. The majority of patients in each group had no concomitant AI diseases: 68.9\% in T1DM group

Table 3 Autoimmune diseases

\begin{tabular}{|c|c|c|c|}
\hline & T1DM $(n=45)$ & LADA $(n=46)$ & $\mathbf{P}$ \\
\hline $\begin{array}{l}\text { Patients with no Al disease, } \\
\text { n (\%) }\end{array}$ & $31(68.9 \%)$ & $30(65.2 \%)$ & 0.710 \\
\hline $\begin{array}{l}\text { Patients with } 1 \mathrm{Al} \text { disease, } \mathrm{n} \\
(\%)\end{array}$ & $9(20.0 \%)$ & $14(30.4 \%)$ & 0.252 \\
\hline $\begin{array}{l}\text { Patients with > } 1 \text { Al diseases, } \\
\text { n (\%) }\end{array}$ & $5(11.1 \%)$ & $2(4.3 \%)$ & 0.267 \\
\hline \multicolumn{4}{|c|}{ Organ-specific autoimmune diseases } \\
\hline $\begin{array}{l}\text { Autoimmune thyroiditis, } \\
\mathrm{n}(\%)\end{array}$ & $12(26.7 \%)$ & $8(17.4 \%)$ & 0.285 \\
\hline Graves disease, $\mathrm{n}(\%)$ & $0(0.0 \%)$ & $4(8.7 \%)$ & 0.117 \\
\hline Atrophic gastritis, n (\%) & $6(13.3 \%)$ & $2(4.3 \%)$ & 0.158 \\
\hline Vitiligo, n (\%) & $1(2.3 \%)$ & $2(4.3 \%)$ & 1.000 \\
\hline Addison disease, $\mathrm{n}(\%)$ & $1(2.3 \%)$ & $0(0.0 \%)$ & 0.495 \\
\hline Lupus, n (\%) & $0(0.0 \%)$ & $1(2.2 \%)$ & 1.000 \\
\hline Autoimmune hepatitis, n (\%) & $1(2.3 \%)$ & $0(0.0 \%)$ & 0.495 \\
\hline Sjogren, n (\%) & $0(0.0 \%)$ & $1(2.2 \%)$ & 1.000 \\
\hline
\end{tabular}

Table 2 Therapeutics on last follow-up

\begin{tabular}{|c|c|c|c|}
\hline Insulin & T1DM $(n=44)$ & LADA $(n=46)$ & $p$ \\
\hline No insulin, $n(\%)$ & $0(0 \%)$ & $4(8.7 \%)$ & 0.117 \\
\hline Basal insulin, $n(\%)$ & $3(6.8 \%)$ & $5(10.9 \%)$ & 0.714 \\
\hline Premixed insulin, $n(\%)$ & $6(13.6 \%)$ & $5(10.9 \%)$ & 0.689 \\
\hline Basal-plus, n (\%) & $1(2.3 \%)$ & $4(8.7 \%)$ & 0.361 \\
\hline Basal-bolus (MDI), n (\%) & $32(72.7 \%)$ & $27(58.6 \%)$ & 0.161 \\
\hline CSII, n (\%) & $2(4.6 \%)$ & $1(2.2 \%)$ & 0.612 \\
\hline Total daily dose (IU), median (IQR) & $40.0(32.0)$ & $33.5(33.0)$ & 0.819 \\
\hline Total daily dose (IU)/Weight $(\mathrm{kg})$, mean ( \pm SD) & $0.58( \pm 0.31)$ & $0.57( \pm 0.39)$ & 0.887 \\
\hline \multicolumn{4}{|l|}{ Non-insulin drugs } \\
\hline Total, n (\%) & $9(20.5 \%)$ & $25(54.3 \%)$ & $0.001^{*}$ \\
\hline Metformin, n (\%) & $7(15.9 \%)$ & $22(47.8 \%)$ & $0.001^{*}$ \\
\hline Sulfonylureas, n (\%) & $0(0 \%)$ & $1(2.2 \%)$ & 1.000 \\
\hline DPP4-i, n (\%) & $5(11.4 \%)$ & $17(37.0 \%)$ & $0.005^{*}$ \\
\hline GLP1-ra, n (\%) & $0(0 \%)$ & $1(2.2 \%)$ & 1.000 \\
\hline SGLT2-i, n (\%) & $0(0 \%)$ & $2(4.3 \%)$ & 0.495 \\
\hline
\end{tabular}

MDI: multiple daily injections; CSII: continuous subcutaneous insulin infusion

* Chi square test 
and $65.2 \%$ in LADA group. T1DM group had more often multiple AI diseases, with 5 patients having more than one condition comparing to 2 patients in LADA group (not statistically significant). The most prevalent condition was AI thyroiditis in both groups, followed by atrophic gastritis, Graves' disease (only present in the LADA group) and vitiligo. None of the patients had the diagnosis of celiac disease.

The percentage of females with AI comorbidities was $66.7 \%$, versus $44.3 \%$ without $(p=0.044)$; there were no gender differences between T1DM and LADA. Female patients with AI thyroiditis corresponded to $70.0 \%$, comparing to $46.5 \%$ without this condition $(p=0.063$, not significant). When we consider T1DM and LADA separately, the percentage of males with AI thyroiditis was $41.7 \%$ and $12.5 \%$, respectively ( $p=0.187$, not significant).

Data regarding diabetic complications at 10 years of diabetes evolution is showed in Table 4. LADA patients had a statistically significant higher prevalence of microvascular complications: $63.3 \%$ comparing to $21.1 \%$ of T1DM patients $(p=0.004)$. This occurred mainly due to diabetic nephropathy, which was present in $53.3 \%$ of LADA and $23.5 \%$ of T1DM $(p=0.047)$. LADA group had a numerically higher proportion of patients with GFR lower than 90 or $60 \mathrm{~mL} / \mathrm{min}$, as well as Albuminuria A3 (not statistically significant). Three patients in LADA group had retinopathy (mild non proliferative diabetic retinopathy in all cases), while no T1DM patient had this complication. LADA also had more often peripheral neuropathy ( 3 patients). T1DM patients had a non-statistically significant trend to a higher prevalence of macrovascular complications.
Clinical and biochemical characteristics of patients at the last evaluation are presented in Table 5. There were no statistically significant differences between both groups in what concerns to A1C, although LADA group showed a trend to higher values. $71.7 \%$ of T1DM patients and $76.1 \%$ of LADA patients had non-optimized metabolic control (A1C over 7\%). LADA group had a numerically higher BMI than T1DM, as well as a higher proportion of overweight and obese patients (not significant). LADA

\begin{tabular}{llll}
$\begin{array}{l}\text { Table } 5 \text { Clinical and biochemical } \\
\text { follow-up }\end{array}$ & & & \\
\hline & T1DM & LADA & $p$ \\
\hline $\begin{array}{l}\text { Diabetes duration at the last } \\
\text { follow-up, years, median (IQR) }\end{array}$ & $8.0(7.5)$ & $11.0(10.5)$ & $0.023^{*}$ \\
A1C (\%), median (IQR) & $7.7(1.9)$ & $8.2(1.7)$ & 0.268 \\
BMI (kg/m²), mean ( \pm SD) & $25.6( \pm 4.3)$ & $27.4( \pm 5.2)$ & 0.086 \\
[n] & {$[42]$} & {$[41]$} & \\
Normal weight, n/total (\%) & $21 / 42(50.0 \%)$ & $13 / 41(31.8 \%)$ & 0.090 \\
Overweight, n/total (\%) & $14 / 42(33.3 \%)$ & $14 / 41(34.1 \%)$ & 0.938 \\
Obesity, n/total (\%) & $7 / 42(16.7 \%)$ & $14 / 41(34.1 \%)$ & 0.067 \\
Hypertension, n/total (\%) & $25 / 46(54.3 \%)$ & $30 / 46(65.2 \%)$ & 0.288 \\
Dyslipidaemia, n/total (\%) & $29 / 46(63.0 \%)$ & $35 / 46(76.1 \%)$ & 0.174 \\
Total cholesterol, mean ( \pm SD) & $183.7( \pm 41.4)$ & $177.6( \pm 41.6)$ & 0.494 \\
HDL cholesterol, median (IQR) & $53.0(26.0)$ & $46.3(14.0)$ & 0.128 \\
LDL cholesterol, mean ( \pm SD) & $113.6( \pm 39.4)$ & $113.6( \pm 37.3)$ & 0.998 \\
Triglycerides, median (IQR) & $85.0(43.0)$ & $93.5(95.0)$ & 0.371 \\
Metabolic syndrome, n/total & $25 / 45(55.6 \%)$ & $38 / 46(82.6 \%)$ & $0.005^{* *}$ \\
$\quad$ (\%) & & & \\
\hline
\end{tabular}

*Independent-samples Mann-Whitney test

**Chi square test

Table 4 Diabetic complications at 10 years of diabetes duration

\begin{tabular}{llcc}
\hline & T1DM & LADA & P \\
\hline Microvascular complications, n/total (\%) & $4 / 19(21.1 \%)$ & $19 / 30(63.3 \%)$ & $0.004^{*}$ \\
Peripheral neuropathy, n/total (\%) & $0 / 19(0.0 \%)$ & $3 / 30(10.0 \%)$ & 0.273 \\
Retinopathy, n/total (\%) & $0 / 18(0.0 \%)$ & $3 / 26(11.5 \%)$ & 0.258 \\
Nephropathy, n/total (\%) & $4 / 17(23.5 \%)$ & $16 / 30(53.3 \%)$ & $0.047^{*}$ \\
GFR (mL/min), median (IQR) & $98.0(19.8)$ & $93.0(23.5)$ & 0.102 \\
[n] & {$[16]$} & {$[28]$} & $13 / 28(46.4 \%)$ \\
GFR<90 mL/min, n/total (\%) & $4 / 16(25.0 \%)$ & $8 / 28(28.6 \%)$ & 0.160 \\
GFR<60 mL/min, n/total (\%) & $2 / 16(12.5 \%)$ & $8.7(16.6)$ & 0.283 \\
Albumin/creatinine ratio (mg/g), median (IQR) & $3.8(12.4)$ & {$[26]$} & 0.149 \\
[n] & {$[15]$} & $3 / 26(11.5 \%)$ & 0.287 \\
Albuminuria A3, n/total (\%) & $0 / 15(0.0 \%)$ & $1 / 31(3.2 \%)$ \\
Macrovascular complications, n/total (\%) & $4 / 20(20.0 \%)$ & $1 / 31(3.2 \%)$ & 0.071 \\
Ischemic Heart Disease, n/total (\%) & $1 / 19(5.3 \%)$ & $0 / 30(0.0 \%)$ \\
Cerebrovascular disease, n/total (\%) & $3 / 19(15.8 \%)$ & $0 / 31(0.0 \%)$ \\
Peripheral artery disease, n/total (\%) & $1 / 20(5.0 \%)$ & 0.053 \\
\hline
\end{tabular}

* Chi square test 
group also had a non-statistically significant higher proportion of patients with hypertension and dyslipidaemia. In what concerns to MetS, $82.6 \%$ of LADA patients and $55.6 \%$ of T1DM patients fulfilled the diagnostic criteria, with a statistically significant difference $(p=0.005)$. This difference remained statistically significant after adjustment for diabetes duration $(\mathrm{OR}=1.10,95 \% \mathrm{CI} 1.02-1.19$, $p=0.014)$.

\section{Discussion}

\section{Baseline characteristics}

AID is a very heterogeneous disease in what concerns to the pathophysiological mechanisms (genetic background, autoimmune process, environmental factors), which leads to a spectrum of clinical profiles with variable degrees of insulin deficiency and insulin resistance $[1,5,9]$. The 2005 IDS diagnostic criteria of LADA [4], although highly applied in clinical practice, raise many questions, such as the lower limit for age (excluding latent autoimmune diabetes of the young [10]) and the subjectivity of the onset of insulin therapy, which is dependent on the physician's decision $[1,4]$. Other scientific societies propose different designations and diagnostic criteria for this clinical entity; this is the case of the Japan Diabetes Society, that considers "Slowly progressive insulin-dependent diabetes mellitus (SPIDDM)", whose diagnostic criteria are (1) the presence of GADA and/or ICA at some time during the disease course and (2) absence of ketosis at onset of DM and no need for insulin treatment to correct hyperglycaemia in the first 3 months after diagnosis [11]. The World Health Organization Classification of Diabetes Mellitus 2019 considers "Slowly evolving, immune mediated diabetes of adults" as a hybrid form of diabetes, although no definitive diagnostic criteria are proposed due to the controversies regarding classification as a separate subtype of diabetes or as a stage of T1DM [12]. Considering their frequent use and the fact that they are easy to apply in clinical practice, we decided to use the IDS criteria in our study. Therefore, we aimed to characterize adult patients (over 30 years old) with AID, comparing patients with classic T1DM and LADA.

In our sample, T1DM patients presented more often with symptoms at diagnosis, which may have justified the early institution of insulin therapy. At the diagnosis, patients with AID may present variable clinical phenotypes, ranging from ketoacidosis to asymptomatic hyperglycaemia that can be controlled with diet alone [5]. In this study, 6 patients in T1DM group were asymptomatic and 21 patients in the LADA group had polyuric/ polydipsic syndrome. Notably, only 2 patients in T1DM group presented with diabetic ketoacidosis, which did not occur in any patient in the LADA group.
The recognition of the autoimmune aetiology of diabetes, defined as the time of measurement of autoantibodies, occurred at the same moment of the diagnosis of diabetes for most patients on T1DM group. Nevertheless, in 5 patients within this group, the measurement of autoantibodies was performed years after diabetes diagnosis (from 3 to 18 years), although these patients were always under insulin therapy. On the other hand, in the LADA group there was a large interval between the onset of diabetes and the establishment of the autoimmune aetiology: median of 5 years (60 months), with a maximum of 37 years. This may be due to the fact that LADA patients are often misdiagnosed as having T2DM [1, 5, 13]. A multicentric Spanish study reported a delay of 3.5 years in LADA confirmation [13], which was similar to our study.

At the diagnosis of the autoimmune aetiology, the T1DM group had a statistically significant lower BMI comparing to LADA, which is supported by literature [9, $14,15]$. This difference may be explained by the important role of lifestyle, leading to insulin resistance in LADA patients [6], as we discuss later. At this same time point, T1DM group had a trend to higher A1C and lower C-peptide, with no statistically significant difference between groups. Other studies reported significant differences in $\mathrm{A} 1 \mathrm{C}$ and $\mathrm{C}$-peptide, being respectively higher and lower in T1DM comparing to LADA $[9,14,15]$. This may be justified by the hypothesis of a more aggressive autoimmune process, with more severe insulinopenia in T1DM patients $[1,2]$.

\section{Autoimmunity}

In what concerns to diabetes-related autoantibodies, T1DM patients had more often multiple positive antibodies and had higher titles of GADA, which has been reported in other studies $[2,5,15,16]$. It has also been reported that among LADA patients, those with higher number of positive antibodies and higher titles of GADA have a "T1DM-like" phenotype, comparing to those with only one positive antibody and lower GADA titles who have a "T2DM-like" phenotype [1, 2, 5, 17]. However, other studies suggest that the presence of GADA, independently of the title, highly increases the risk of progression to insulin dependence, comparing to T2DM [18].

Autoantibodies do not seem to be the key pathogenic factor in AID, but rather a marker of a process that appears to be mediated by immune cell response [1] LADA patients share genetic variants in human leukocyte antigen (HLA) complex with T1DM patients, which confer susceptibility to AID [16, 19, 20]. Nevertheless, in T1DM patients the autoimmune process is more aggressive, leading to severe beta-cell destruction, insulinopenia and risk of ketosis $[1,6]$. 
T2DM-risk genetic variants are not so common in LADA patients $[19,20]$ and the pathological mechanisms of beta-cell failure seem different between LADA and T2DM [16]. However, these two populations seem to share the unhealthy lifestyle, which increases the risk of overweight, increased adiposity and insulin resistance $[6,20]$. In fact, in our sample LADA patients had more often MetS comparing to T1DM patients. Therefore, in LADA both insulin deficiency and resistance play important roles in the pathogenesis $[5,6,9]$.

In our sample, more than two-thirds of patients in both groups did not have other autoimmune diseases. Nevertheless, the few T1DM who had other autoimmune disorders had more often multiple conditions, which can be a sign of a more aggressive autoimmune process $[1,5,6]$. The most frequent comorbidity was thyroid autoimmune disorder. Interestingly, no patient had the diagnosis of celiac disease, which is frequently associated to childhood T1DM [21].

In this study, female patients had more often other AI conditions, namely AI thyroiditis. Zampetti et al. [17] reported a higher prevalence of thyroid antibodies in male LADA patients with higher titles of GADA. In our sample, the percentage of male patients with AI thyroiditis was superior in T1DM group, reinforcing the concept that a more intense autoimmune profile increases the risk of AI thyroiditis in male patients.

\section{Diabetes treatment}

In what concerns to diabetes treatment at the last evaluation, in our sample all T1DM patients were under insulin therapy, mainly basal-bolus regimen. Most LADA patients were under basal-bolus therapy: a total of $58.6 \%$, which seems lower than other series [13]. The mean TDD adjusted for weight was very similar between both groups $(0.58 \mathrm{IU} / \mathrm{kg}$ in T1DM and $0.57 \mathrm{IU} / \mathrm{kg}$ in LADA).

Although LADA has distinct pathophysiological mechanisms of disease comparing to T1DM (less pronounced insulinopenia and significant insulin resistance), with the progression of the disease most patients eventually need insulin therapy $[1,3,13]$. Most studies on LADA compare these patients with T2DM and describe a faster progression to insulin therapy, mainly in patients with higher GADA titles $[1,2,5]$. Nevertheless, in our sample four LADA patients (8.7\%) were not under insulin treatment, with no episodes of ketosis (three of them with good glycaemic control). On the other hand, $54.3 \%$ of LADA patients were under noninsulin antidiabetics (comparing to $20.5 \%$ of T1DM group), which seems lower than other series [13, 22].

\section{Metabolic control and diabetes complications}

In the field of diabetes complications, at 10 years of diabetes duration, we report a significant higher frequency of microvascular complications in LADA, especially due to diabetic nephropathy. The slow progression of disease in LADA may be associated with asymptomatic hyperglycaemia before diagnosis, which together with other metabolic risk factors, leads to a continuous micro and macrovascular damage $[1,15]$. There are reports in the literature of LADA patients with established micro and macrovascular complications in the first year after diabetes diagnosis [15]. In our study, patients in the LADA group showed a trend to have higher weight, HbA1c and blood pressure values, and this may have contributed to the increased frequency of nephropathy in this group.

There are not many studies comparing long term diabetes complications between T1DM and LADA. For identical disease duration, the frequency of albuminuria and chronic kidney disease seems to be identical between LADA and T2DM, being higher in these groups comparing to T1DM [22]. However, a post hoc analysis from UKPDS study showed a lower risk of microvascular complications at diabetes onset in LADA patients comparing to T2DM, followed by a higher risk after 9 years of disease due to worse glycaemic control [23]. In what concerns to cardiovascular disease, it seems to occur at a higher frequency in T2DM, comparing to LADA and T1DM [22, 24]. Our study showed a non-statistically significant trend to a higher proportion of T1DM patients with macrovascular complications. Literature data regarding differences between LADA and T1DM in this field are conflicting: Luk et al. report similar long-term frequencies in cardiovascular outcomes [22], while Wod et al. report a lower prevalence in LADA [24].

In what concerns to glycaemic control on last followup, LADA patients had a non-statistically significant higher A1C (8.2\% comparing to $7.7 \%$ in T1DM) and a higher percentage of patients with A1C over 7\%. A possible bias is the longest median duration of disease in LADA group (11 years versus 8 years in T1DM). Nevertheless, Luk et al. reported identical mean A1C values for T1DM and LADA (8.5\% and 8.4\%), after a median duration of diabetes of 8 and 6 years respectively [22]. On the other hand, LADA group had a statistically significant higher proportion of patients with MetS: $82.6 \%$ comparing to $55.6 \%$ of T1DM patients. This difference between groups remained significant after adjustment for diabetes duration. The proportion of MetS in LADA is comparable to its prevalence in T2DM patients [25]. When we consider isolated components of MetS, LADA patients had a non-statistically significant trend to have more often BMI over $25 \mathrm{~kg} / \mathrm{m}^{2}$, hypertension and dyslipidaemia, which confirms other published results [21]. 
These differences reflect the important role of adiposity and insulin resistance in the pathogenesis of LADA and its related complications $[1,3,6]$.

\section{Strengths and limitations on this study}

This is a retrospective study with the limitations related to this kind of design: the unavailability of all data in patients' files and the non-uniform diagnostic and therapeutic strategies. The non-matching of patients in what concerns to disease duration in the groups may lead to some difficulties in interpreting the results. Nonetheless, the patients included in the study represent a "realworld" sample of diabetic patients followed on a tertiary care centre. To the best of our knowledge, few studies compared T1DM and LADA patients, especially with long duration of disease.

\section{Conclusions}

Our study showed that AID is a very heterogeneous condition, with a clinical presentation that may range from asymptomatic hyperglycaemia to diabetic ketoacidosis. Patients with classic T1DM presented more often with symptoms at diagnosis, lower BMI and higher number of autoantibodies, which may be related to a more aggressive autoimmune process. This symptomatic presentation is probably the main factor leading to an early institution of insulin therapy. Patients with LADA developed more frequently microvascular complications and particularly nephropathy, also having a trend to a higher prevalence of peripheral neuropathy and retinopathy. This may be related with a more insidious nature of the disease, with higher glucose exposure, as well as with higher levels of insulin resistance and its associated comorbidities. LADA patients also had a higher prevalence of metabolic syndrome, which strengths the role of adiposity and insulin resistance in the pathophysiology of this type of diabetes and its complications.

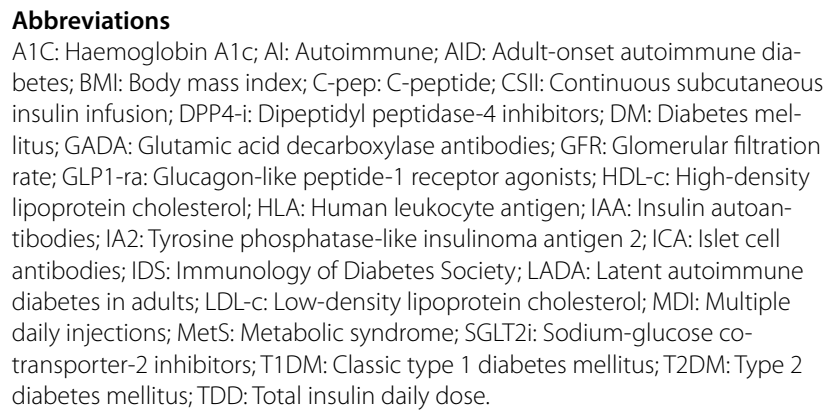
betes; BMI: Body mass index; C-pep: C-peptide; CSII: Continuous subcutaneous insulin infusion; DPP4-i: Dipeptidyl peptidase-4 inhibitors; DM: Diabetes mellitus; GADA: Glutamic acid decarboxylase antibodies; GFR: Glomerular filtration rate; GLP1-ra: Glucagon-like peptide-1 receptor agonists; HDL-c: High-density lipoprotein cholesterol; HLA: Human leukocyte antigen; IAA: Insulin autoantibodies; IA2: Tyrosine phosphatase-like insulinoma antigen 2; ICA: Islet cell antibodies; IDS: Immunology of Diabetes Society; LADA: Latent autoimmune diabetes in adults; LDL-c: Low-density lipoprotein cholesterol; MDI: Multiple daily injections; MetS: Metabolic syndrome; SGLT2i: Sodium-glucose cotransporter-2 inhibitors; T1DM: Classic type 1 diabetes mellitus; T2DM: Type 2 diabetes mellitus; TDD: Total insulin daily dose.

\section{Acknowledgements}

Not applicable.

\section{Authors' contributions}

$L F$, JS and $M M$ were involved in the conception and design of the work. $L F, D C$ and JF conducted data collection. LF performed data analysis and the article drafting. JS and MM critically reviewed the manuscript. IP oversaw the creation of the manuscript. All authors read and approved the final manuscript.

\section{Funding}

This research did not receive any specific grant from funding agencies in the public, commercial, or not-for-profit sectors.

\section{Availability of data and materials}

The datasets used and/or analysed during the current study are partially included within the article. Complete datasets are available from the corresponding author on reasonable request.

\section{Ethics approval and consent to participate}

This study was approved by the local review boards (Centro Hospitalar e Universitário de Coimbra) and all participants signed the consent to use their data for scientific purpose.

\section{Consent for publication}

Consent for publication of raw data not obtained, but dataset is fully anonymous.

\section{Competing interests}

The authors declare that they have no competing interests.

\section{Author details}

${ }^{1}$ Endocrinology, Diabetes and Metabolism Department, Centro Hospitalar e Universitário de Coimbra, Coimbra, Portugal. ${ }^{2}$ Faculty of Medicine, University of Coimbra, Coimbra, Portugal. ${ }^{3}$ Clinical Pathology Department, Centro Hospitalar e Universitário de Coimbra, Coimbra, Portugal. ${ }^{4}$ Instituto de Investigação e Inovação em Saúde (I3S)/Institute of Pathology and Immunology of the University of Porto (Ipatimup), Porto, Portugal.

Received: 1 September 2020 Accepted: 21 November 2020

Published online: 03 December 2020

\section{References}

1. Buzzetti R, Zampetti S, Maddaloni E. Adult-onset autoimmune diabetes: current knowledge and implications for management. Nat Rev Endocrinol. 2017;13(11):674-86. https://doi.org/10.1038/nrendo.2017.99.

2. Sørgjerd EP. Type 1 diabetes-related autoantibodies in different forms of diabetes. Curr Diabetes Rev. 2019;15(3):199-204. https://doi. org/10.2174/1573399814666180730105351.

3. Kumar A, de Leiva A. Latent autoimmune diabetes in adults (LADA) in Asian and European populations. Diabetes Metab Res Rev. 2017. https:// doi.org/10.1002/dmrr.2890.

4. Fourlanos S, Dotta F, Greenbaum CJ, Palmer JP, Rolandsson O, Colman PG, Harrison LC. Latent autoimmune diabetes in adults (LADA) should be less latent. Diabetologia. 2005;48(11):2206-12.

5. Laugesen E, Østergaard JA, Leslie RD. Danish Diabetes Academy Workshop and Workshop Speakers. Latent autoimmune diabetes of the adult: current knowledge and uncertainty. Diabet Med. 2015;32(7):843-52. https://doi.org/10.1111/dme.12700.

6. Carlsson S. Etiology and Pathogenesis of Latent Autoimmune Diabetes in Adults (LADA) Compared to Type 2 Diabetes. Front Physiol. 2019;26(10):320. https://doi.org/10.3389/fphys.2019.00320.

7. Alberti KG, Eckel RH, Grundy SM, et al. Harmonizing the metabolic syndrome: a joint interim statement of the International Diabetes Federation Task Force on Epidemiology and Prevention; National Heart, Lung, and Blood Institute; American Heart Association; World Heart Federation; International Atherosclerosis Society; and International Association for the Study of Obesity. Circulation. 2009;120(16):1640-5. https://doi. org/10.1161/CIRCULATIONAHA.109.192644.

8. Kidney Disease: Improving Global Outcomes (KDIGO) CKD Work Group. KDIGO. clinical practice guideline for the evaluation and management of chronic kidney disease. Kidney Int Suppl. 2012;2013(3):1-150. 
9. Yang L, Liu X, Liang H, et al. Pathophysiological characteristics in patients with latent autoimmune diabetes in adults using clamp tests: evidence of a continuous disease spectrum of diabetes. Acta Diabetol. 2019;56:121724. https://doi.org/10.1007/s00592-019-01387-6.

10. Stene LC, Barriga K, Hoffman M, et al. Normal but increasing hemoglobin A1c levels predict progression from islet autoimmunity to overt type 1 diabetes: diabetes Autoimmunity Study in the Young (DAISY). Pediatr Diabetes. 2006;7(5):247-53.

11. Tanaka S, Ohmori M, Awata T, et al. Diagnostic criteria for slowly progressive insulin-dependent (type 1) diabetes mellitus (SPIDDM) (2012): report by the Committee on Slowly Progressive Insulin-Dependent (Type 1) Diabetes Mellitus of the Japan Diabetes Society. Diabetol Int. 2015;6:1-7. https://doi.org/10.1007/s13340-014-0199-2.

12. Classification of diabetes mellitus. Geneva: World Health Organization; 2019. Licence: CC BY-NC-SA 3.0 IGO.

13. Arranz Martín A, Lecumberri Pascual E, Brito Sanfiel MÁ, et al. Clinical and metabolic profile of patients with latent autoimmune diabetes in adults in specialized care in Madrid. Endocrinol Diabetes Nutr. 2017;64(1):34-9. https://doi.org/10.1016/j.endinu.2016.09.001.

14. Pipi E, Marketou M, Tsirogianni A. Distinct clinical and laboratory characteristics of latent autoimmune diabetes in adults in relation to type 1 and type 2 diabetes mellitus. World J Diabetes. 2014;5(4):505-10. https://doi. org/10.4239/wjd.v5.i4.505.

15. Zaharia OP, Bobrov P, Strassburger K, et al. Metabolic characteristics of recently diagnosed adult-onset autoimmune diabetes mellitus. J Clin Endocrinol Metab. 2018;103(2):429-37. https://doi.org/10.1210/jc.201701706.

16. Nishimura A, Matsumura K, Kikuno S, Nagasawa K, Okubo M, Mori Y, Kobayashi T. Slowly progressive type 1 diabetes mellitus: current knowledge and future perspectives. Diabetes Metab Syndr Obes. 2019;28(12):246177. https://doi.org/10.2147/DMSO.S191007.

17. Zampetti S, Capizzi M, Spoletini M, Campagna G, Leto G, Cipolloni L, Tiberti C, Bosi E, Falorni A, Buzzetti R, NIRAD Study Group. GADA titerrelated risk for organ-specific autoimmunity in LADA subjects subdivided according to gender (NIRAD study 6). J Clin Endocrinol Metab. 2012;97(10):3759-65. https://doi.org/10.1210/jc.2012-2037.

18. Tanaka S, Okubo M, Nagasawa K, Takizawa S, Ichijo M, Ichijo S, Kaneshige M, Aida K, Shimura H, Mori Y, Kobayashi T. Predictive value of titer of GAD antibodies for further progression of beta cell dysfunction in slowly progressive insulin-dependent (type 1) diabetes (SPIDDM). Diabetol Int. 2015;7(1):42-52. https://doi.org/10.1007/s13340-015-0211-5.
19. Mishra R, Chesi A, Cousminer DL, et al. Relative contribution of type 1 and type 2 diabetes loci to the genetic etiology of adult-onset, non-insulinrequiring autoimmune diabetes. BMC Med. 2017;15(1):88. https://doi. org/10.1186/s12916-017-0846-0.

20. Hjort R, Löfvenborg JE, Ahlqvist $\mathrm{E}$, et al. Interaction between overweight and genotypes of HLA, TCF7L2, and FTO in relation to the risk of latent autoimmune diabetes in adults and type 2 diabetes. J Clin Endocrinol Metab. 2019;104(10):4815-26. https://doi.org/10.1210/jc.2019-00183.

21. Shahramian I, Bazi A, Sargazi A. An overview of Celiac disease in childhood type 1 diabetes. Int J Endocrinol Metab. 2018;16(3):e66801. https:// doi.org/10.5812/ijem.66801.

22. Luk AOY, Lau ESH, Lim C, et al. Diabetes-related complications and mortality in patients with young-onset latent autoimmune diabetes: a 14-year analysis of the prospective Hong Kong Diabetes Register. Diabetes Care. 2019;42(6):1042-50. https://doi.org/10.2337/dc18-1796.

23. Maddaloni E, Coleman RL, Agbaje O, Buzzetti R, Holman RR. Time-varying risk of microvascular complications in latent autoimmune diabetes of adulthood compared with type 2 diabetes in adults: a post hoc analysis of the UK Prospective Diabetes Study 30-year follow-up data (UKPDS 86). Lancet Diabetes Endocrinol. 2020;8(3):206-15. https://doi.org/10.1016/ S2213-8587(20)30003-6.

24. Wod M, Thomsen RW, Pedersen L, Yderstraede KB, Beck-Nielsen $H_{\text {, }}$ Højlund K. Lower mortality and cardiovascular event rates in patients with Latent Autoimmune Diabetes In Adults (LADA) as compared with type 2 diabetes and insulin deficient diabetes: a cohort study of 4368 patients. Diabetes Res Clin Pract. 2018;139:107-13. https://doi. org/10.1016/j.diabres.2018.02.042.

25. lanne-Parikka P, Eriksson JG, Lindström J, Hämäläinen $H$, KeinänenKiukaanniemi S, Laakso M, Louheranta A, Mannelin M, Rastas M, Salminen V, Aunola S, Sundvall J, Valle T, Lahtela J, Uusitupa M, Tuomilehto J, Finnish Diabetes Prevention Study Group. Prevalence of the metabolic syndrome and its components: findings from a Finnish general population sample and the Diabetes Prevention Study cohort. Diabetes Care. 2004;27(9):2135-40. https://doi.org/10.2337/diacare.27.9.2135.

\section{Publisher's Note}

Springer Nature remains neutral with regard to jurisdictional claims in published maps and institutional affiliations.
Ready to submit your research? Choose BMC and benefit from:

- fast, convenient online submission

- thorough peer review by experienced researchers in your field

- rapid publication on acceptance

- support for research data, including large and complex data types

- gold Open Access which fosters wider collaboration and increased citations

- maximum visibility for your research: over 100M website views per year

At BMC, research is always in progress.

Learn more biomedcentral.com/submissions 